\title{
Le grand tunnel hydrodynamique (GTH)
}

\author{
Y. Lecoffre Bassin d'Essais des Carènes, le Val de Reuil \\ P. Chantrel CERG-Alsthom, Le Pont de Claix \\ J. Teiller ACB-Alsthom, Nantes
}

\section{Introduction}

Afin de satisfaire aux exigences de plus en plus rigoureuses en matière de recherche hydrodynamique navale, un nouveau centre d'essai a été construit au Val de Reuil, près de Rouen à environ $100 \mathrm{~km}$ à l'ouest de Paris. Le maître d'ouvrage est la Direction des constructions navales, Bassin des Carènes, le conducteur d'opération est l'ETCA à Arcueil, l'exploitant du centre sera le Bassin des Carènes.

Ce centre dispose de deux tunnels de cavitation. Le premier, connu sous le sigle PTH (Petit Tunnel Hydrodynamique) est une installation de taille moyenne, dont la section d'essai en surface libre mesure $0,6 \times 0,5 \times 3 \mathrm{~m}$. La vitesse d'écoulement maximale y est de $15 \mathrm{~m} / \mathrm{s}$. Le PTH se trouvait auparavant au Bassin des Carènes à Paris, et a été entièrement reconstruit au Val de Reuil en janvier 1987.

Le second, le GTH (Grand Tunnel Hydrodynamique) construit par Alsthom, est un grand tunnel comportant deux sections d'essai parallèles. La veine en surface libre (VSL) mesure $2 \times 1,35 \times 10 \mathrm{~m}$; la vitesse maximale y est de $12 \mathrm{~m} / \mathrm{s}$. La veine fermée (VF) a une section carrée de $1,14 \mathrm{~m}$ de côté et une longueur de $6 \mathrm{~m}$. La vitesse peut atteindre $20 \mathrm{~m} / \mathrm{s}$.

Le PTH a été décrit en [2], et un aperçu général du GTH se trouve en [1]. Nous décrirons ici dans le détail les principaux éléments du GTH et traiterons des performances de ce tunnel [6].

\section{Architecture du GTH}

Le GTH est entièrement logé dans un bâtiment de $85 \mathrm{~m}$ de long, de $30 \mathrm{~m}$ de large et de $25 \mathrm{~m}$ de haut. La figure 1 représente un dessin du tunnel proprement dit. Le volume d'eau total est de $3600 \mathrm{~m}^{3}$. La cuve aval (CA) est utilisée pour évacuer l'air produit ou injecté dans la section d'essai. Elle a un volume total de $1600 \mathrm{~m}^{3}$, et peut évacuer l'air d'émulsions dont les taux de vides peuvent atteindre $10 \%$. D'autre part, les bulles supérieures à $100 \mu \mathrm{m}$ sont toutes éliminées dans la cuve aval, même au débit maximum de $32,4 \mathrm{~m}^{3} / \mathrm{s}$.

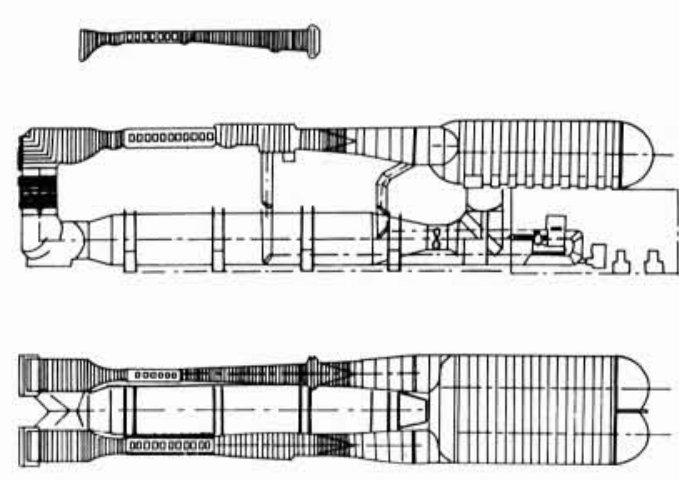

1. Structure générale du GTH.

\section{The Great Hydrodynamic Tunnel (GTH).}

Known as the GTH (Great Hydrodynamic Tunnel), the recently built French cavitation tunnel intended for research concerning naval hydrodynamics is situated in Val de Reuil, in the West of Paris. This installation comprises two parallelly placed test sections, one closed and the other in open space. The maximum velocities of flow are respectively 20 and $12 \mathrm{~m} / \mathrm{s}$. This article describes the main elements of the tumnel. The original control systems of microbubbles and energy recuperation of flows in open space are set out in more detail. 
Les concentrations en germes de cavitation sont contrôlées automatiquement par des générateurs de microbulles, par des systèmes de mesure, et par des dispositifs de dégazage intégrés, situés à l'amont des convergents. Un grand résorbeur de $5 \mathrm{~m}$ de diamètre complète la fonction de la cuve aval. Aucun germe ne retourne dans la section d'essai après que l'eau ait accompli un tour complet de tunnel. Pour réduire le bruit d'écoulement, les vitesses de l'eau sont partout inférieures à $2,5 \mathrm{~m} / \mathrm{s}$, à l'exception, bien entendu, des sections d'essai et des pompes.

Les deux sections d'essai sont disposées en parallèle et fonctionnent de manière indépendante mais non simultanée. Ceci a légèrement accru les coûts de construction, par rapport à des sections d'essai interchangeables, mais l'analyse a montré que cette disposition supplémentaire serait rapidement rentabilisée par l'augmentation du temps d'essai effectif qui en résulte.

\section{Principaux éléments du tunnel}

\section{Cuve aval (CA)}

La cuve aval sert à éliminer l'air libre avant qu'il n'atteigne la pompe, et à contrôler le niveau général de pression dans le tunnel. Il s'agit d'un séparateur gravitaire à haute efficacité. La cuve aval peut éliminer les bulles millimétriques contenues dans des émulsions de taux de vide égal à $10 \%$, tout en séparant en même temps toutes les bulles supérieures à $100 \mu \mathrm{m}$, ceci pour des débits allant jusqu'à $32,4 \mathrm{~m}^{3} / \mathrm{s}$. Une description complète du système se trouve en [3]. L'eau provenant de l'une des sections d'essai passe à travers les diffuseurs 1 et le double coude 2, tels qu'illustrés à la figure 2. Elle se divise ensuite en deux flux $(3,4)$, qui entrent dans les séparateurs 6 par la grille 5 , conçue pour redresser l'écoulement. A la sortie du séparateur, l'eau accélère à nouveau et est dirigée sur la pompe par un passage prévu en dessous des séparateurs.

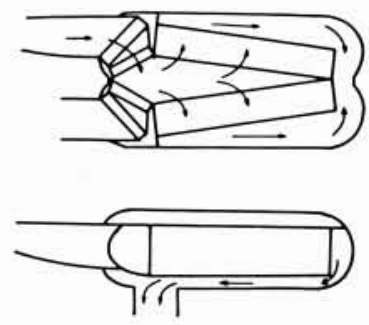

2. Ecoulement interne dans la cuve aval.

La distribution de la vitesse dans la cuve aval devant être aussi uniforme que possible, l'écoulement a été étudié au préalable sur une maquette à l'échelle $1 / 10$, dans laquelle les répartitions de vitesse ont été mesurées. Ces essais ont entraîné quelques modifications dans la conception de la cuve, qui ont permis de supprimer les écoulements secondaires inacceptables et les phénomènes de recirculation.

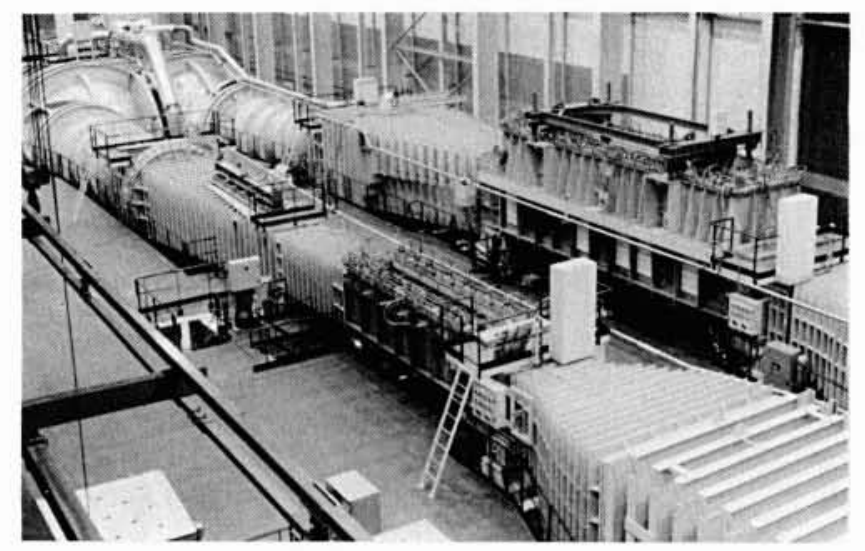

3. Vue générale du GTH.

Des essais en grandeur réelle ont été effectués sur les trois étages du séparateur (fig. 4). Le premier étage est un séparateur à plaques parallèles (1), conçu pour évacuer les bulles millimétriques.

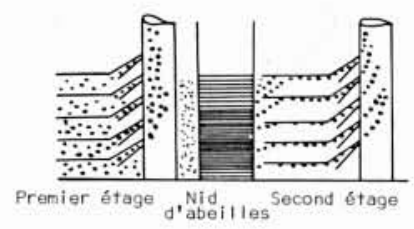

4. Séparateur gravitaire.

Les bulles collectées dans le sommet des plaques situées à la base du séparateur doivent encore s'élever de $4,5 \mathrm{~m}$ pour atteindre le niveau supérieur de la cuve. Pour permettre ce processus, elles sont collectées dans une série de cheminées verticales, dont la conception découle d'essais au réel systématiques menés sur les écoulements à l'intérieur et autour desdites cheminées.

Les microbulles - inférieures à $500 \mu \mathrm{m}$ - ne peuvent être décantées dans le premier étage, du fait de la turbulence. Elles passent à travers un nid d'abeilles 2, dans lequel l'écoulement est laminaire et où se produit un processus complexe de séparation gravitaire et de coalescence. La taille des bulles sortant du nid d'abeilles est supérieure à $1 \mathrm{~mm}$. Le troisième étage est identique au premier, et le système complet permet d'éliminer les bulles supérieures à $100 \mu \mathrm{m}$.

\section{Résorbeur}

Les bulles inférieures à $100 \mu \mathrm{m}$ doivent être dissoutes avant d'atteindre la section d'essai. Il est bien connu qu'un résorbeur placé au fond du tunnel augmente le processus de dissolution, qui se produit dans l'ensemble du tunnel. Le résorbeur du GTH a $30 \mathrm{~m}$ de long et $5 \mathrm{~m}$ de diamètre. Le temps de séjour moyen, pour le débit maximum, est de $18,4 \mathrm{~s}$.

Le CERG a mené des expériences dans le but de déterminer le temps moyen nécessaire en situation pratique pour éliminer complètement les bulles. Un 
modèle mathématique de comportement de bulle a ainsi été élaboré. Pour une distribution de bulles donnée dans la section d'essai, qui peut dépendre des conditions de fonctionnement du tunnel et de la maquette d'essai, le modèle mathématique prend en compte les effets de la géométrie du tunnel, la performance de la cuve aval et la capacité de résorption des bulles. L'histogramme des bulles produit par la section d'essai se modifie lorsque l'écoulement se déplace le long du tunnel, et cette modification peut être calculée pour toutes les conditions de fonctionnement.

\section{Pompes}

La pompe principale du tunnel est une pompe axiale Alsthom-Rateau, avec un rotor à dix pales et une puissance maximale de $1800 \mathrm{~kW}$. Alsthom-Rateau a conçu cette pompe pour alimenter indifféremment l'une ou l'autre des veines d'essais. Elle a fait l'objet d'essais très complets pour des débits simulés de 26 et $32,4 \mathrm{~m}^{3} / \mathrm{s}$, avec des hauteurs manométriques de 6,0 et $4,5 \mathrm{~m}$ respectivement. Ces essais sur maquette ont montré que la pompe, dans ces deux conditions de fonctionnement, ne présentait aucune cavitation, ce qui est essentiel pour obtenir un fonctionnement silencieux du tunnel.

La pompe auxiliaire, une pompe hélico-centrifuge de $300 \mathrm{~kW}$, également fabriquée par Alsthom-Rateau, est utilisée pour injecter l'eau écrémée de la surface libre dans la cuve aval. Cette pompe ne présente également aucune cavitation.

\section{Coudes}

Tous les coudes sont équipés d'ailettes de $1 \mathrm{~m}$ de corde qui ont été conçues en utilisant des calculs potentiel 2D. La distance entre les aubes adjacentes est d'environ un tiers de leur longueur. Leurs bords d'attaques sont elliptiques, et leurs bords de fuite sont chanfreinés. Des essais acoustiques ont été conduits sur maquette, pour déterminer de manière précise le nombre de Strouhal critique des aubes. Ces essais ont également servi à calculer la distance entre les raidisseurs disposés perpendiculairement aux aubes directrices. Ces raidisseurs ont, bien sûr, été réalisés avec des bords d'attaque elliptiques et des bords de fuite chanfreinés.

\section{Nids d'abeille}

Les nids d'abeille servant à améliorer la qualité de l'écoulement dans la section d'essai, sont situés en amont

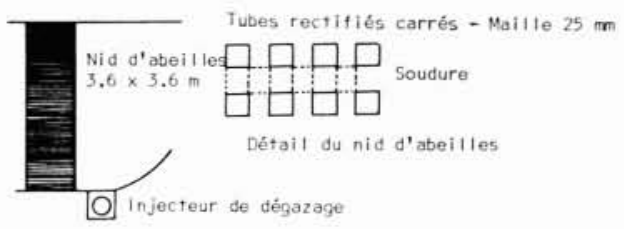

5. Nids d'abeille du GTH. de chaque convergent. Ils suppriment les turbulences de basse fréquence et, grâce à la perte de pression à travers les canaux, augmentent l'homogénéité de la répartition de vitesse axiale.

Les nids d'abeille du GTH ont une longueur de $800 \mathrm{~mm}$, et sont situés dans des canalisations carrées de $3,6 \times 3,6 \mathrm{~m}$. Ils se composent de tubes rectifiés, soudés bord à bord, de $25 \times 25 \mathrm{~mm}$. Chaque nid d'abeille comprend 10000 tubes, représentant une longueur totale de $8 \mathrm{~km}$ (fig. 5).

\section{Convergents}

Les taux de convergence sont de 10 pour la VF et de 4,8 pour la VSL. La répartition de vitesse dans les sections d'essai est fonction de la répartition à l'amont des convergents. Si l'on considère $V_{0}$ comme étant la vitesse moyenne à l'amont de la contraction et $V_{s}$ la vitesse moyenne dans la section d'essai, une non-homogénéité de vitesse dans le plan $O$ (fig. 6) peut être définie de la façon suivante :

$$
V_{0}^{\prime}=V_{0}(1+\varepsilon) \text {. }
$$

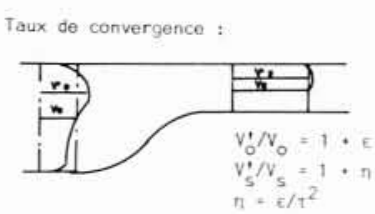

6. Réduction de la non-homogénéité de vitesse par effet de convergence.

La non-homogénéité de vitesse sur la ligne de courant associée dans la section d'essai est facile à calculer. Si l'on considère $V_{s}^{\prime}$ comme étant la vitesse dans la section d'essais, il est possible de la définir de la façon suivante :

$$
V_{s}^{\prime}=V_{s}(1+\eta) \text {. }
$$

Nous pouvons alors appliquer l'équation de Bernoulli :

$$
P_{0}+0,5 \rho V_{0}^{2}=P_{s}+0,5 \rho V_{s}^{2}
$$

et

$$
P_{0}+0,5 \rho V_{0}^{2}(1+\varepsilon)^{2}=P_{s}+0,5 \rho V_{s}(1+\eta)^{2}
$$

étant le taux de contraction, on trouve :

$$
\eta=\varepsilon\left(1 / \tau^{2}\right) .
$$

En conséquence, si les vitesses de la section d'essai doivent être uniformes à mieux que $1 \%$, les non-homogénéités de vitesse à l'amont du convergent dans le cas de la VSL, par exemple, doivent être inférieures à $23 \%$.

Une méthode hybride de calcul 2D-3D a été utilisée pour étudier le convergent. La configuration de base utilisée était un jet libre 2D. On a choisi une ligne de courant interne approximée par un polynôme de puissance 5. La contraction (fortement asymétrique) qui en a 
résulté, a ensuite été calculée avec un code 3D. Des calculs de couche limite basés sur la méthode de von Karman ont été effectués. Les deux convergents ont ensuite été testés sur des maquettes à paroi transparente à l'échelle $1 / 6$ pour vérifier qu'aucune cavitation ne s'y produisait à la valeur minimale de $\sigma$ requise.

\section{Divergents}

Les divergents sont l'un des éléments critiques des tunnels de cavitation. Du fait des courbures locales dans la région de plus faible pression, ils sont susceptibles de produire une cavitation pour de faibles valeurs du $\sigma$ de fonctionnement. Les diffuseurs peuvent également engendrer des gradients de pression adverses entraînant des instabilités de basse fréquence.

La perte de pression des diffuseurs doit être aussi faible que possible et il est bien connu qu'une perte de pression minimale intervient avec un diffuseur de $7^{\circ}$. De même, on sait que la cavitation est inévitable avec une telle conception. En tenant compte de ce qui précède, la longueur du diffuseur et son coût d'ensemble doivent être minimisés.

Les diffuseurs GTH sont un compromis entre ces diverses exigences conflictuelles. Ils se composent de plusieurs éléments de diffusion, avec un angle équivalent moyen de $5^{\circ}$. Le dernier élément situé juste à l'amont de la cuve aval est un diffuseur double, avec un angle équivalent de $7^{\circ}$. Les calculs de diffuseurs par NavierStokes ont montré qu'aucune séparation ne se produit. De même, les tests sur des maquettes de sections d'essai à parois transparentes à échelle $1 / 6$ ont pu mettre en évidence l'absence totale de cavitation, quel que soit le nombre de cavitation dans la section d'essai, le minimum étant de 0,065 dans la veine fermée.

\section{Caractéristiques particulières}

\section{Dégazage}

Avec les procédures de dégazage traditionnelles, il faudrait au moins une semaine pour supprimer les gaz dissous dans les $3600 \mathrm{~m}^{3}$ d'eau du GTH. On a jugé que le fonctionnement optimal du tunnel exigeait que l'eau soit dégazée à une saturation de $20 \%$ dans un délai de 60 min environ. Un système de dégazage spécial s'avérait ainsi nécessaire. Les dégazeurs traditionnels furent jugés trop coûteux, et il fut, par conséquent, décidé d'incorporer un système de dégazage Lecoffre à grande vitesse [4]. La vitesse de transfert de masse sur une interface gázliquide est donnée par :

$$
\mathrm{d} m / \mathrm{d} t=K L A H\left(P_{\text {sat }}-P\right)
$$

où

$$
\begin{aligned}
\mathrm{d} m / \mathrm{d} t= & \text { vitesse de masse du transfert de gaz } \\
K L & =\text { coefficient de transfert de masse } \\
A \quad & =\text { aire de l'interface } \\
H \quad & \text { constante d'Henry } \\
P_{\text {sat }} \quad= & \text { pression de saturation d'équilibre des espèces } \\
& \text { considérées }
\end{aligned}
$$

$P \quad=$ pression partielle des espèces dans la phase gazeuse.

La vitesse de transfert maximale nécessitant que $K L A$ soit aussi élevé que possible, une surface d'échange extrêmement grande est créée dans le GTH, en injectant des microbulles juste en amont du convergent. La figure 7 montre les vitesses de transfert obtenues avec et sans injection de microbulles, pendant les essais sur maquette menés dans le tunnel de cavitation TH8 du CERG-ACB. Les essais réalisés au GTH confirment les valeurs estimées des temps de dégazage.

Le générateur de microbulles du GTH exige de l'eau ayant une teneur en gaz relativement élevée. Elle est produite par le système générateur de germes. L'ensemble du système comprenant les générateurs de germes et le système de dégazage fait ainsi partie intégrante du tunnel.

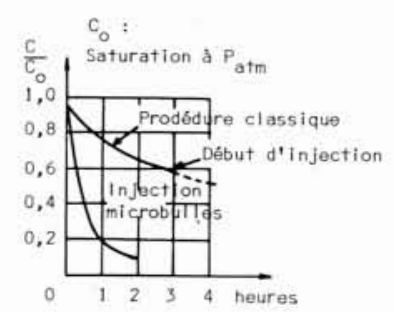

7. Dégazage avec et sans injection de microbulles.

\section{Générateurs de germes}

L'étude du CERG sur les générateurs de germes qui utilisent de l'eau à forte saturation, est décrite en [4]. Le fonctionnement du GTH nécessite une concentration de germes, uniformément répartie, de 30 germes par $\mathrm{cm}^{3}$ au débit maximum. De nouveaux générateurs ont été mis au point à cette fin et des essais de maquette ont montré qu'ils pouvaient produire $10^{9}$ germes/s, pour un débit d'eau saturée de $20 \mathrm{~b} / \mathrm{s}$.

Les générateurs de germes du GTH forment une grille carrée régulière de maille $600 \mathrm{~mm}$ située dans une section verticale en amont des convergents. Ils peuvent produire, soit une concentration de germes homogène dans la section d'essai, soit une distribution avec concentration renforcée le long de l'axe. Le degré de saturation et la pression de l'eau sursaturée sont contrôlés automatiquement, afin que chaque distribution de germes souhaitée puisse être produite à la demande, à partir du poste de commande du tunnel.

\section{Mesures de germes}

Les germes sont mesurés par la méthode du Venturi (Oldenziel, Lecoffre), par diffusion optique [5] ou par la méthode optique des phases. Comme le montre la figure 8, l'eau échantillonnée juste en amont du nid d'abeilles peut passer à travers l'un ou l'autre des deux premiers systèmes. Après la mesure, l'eau est renvoyée dans le tunnel par l'intermédiaire d'une pompe à vitesse variable. Le système Venturi est utilisé directement pour 
de faibles concentrations, jusqu'à 1 germe $/ \mathrm{cm}^{3}$. On utilise le Venturi muni d'un système de dilution pour mesurer des concentrations pouvant atteindre 100 bulles $/ \mathrm{cm}^{3}$ [7]. Il est prévu d'utiliser la méthode de diffusion pour des concentrations plus importantes, ou pour déterminer la distribution de rayon des microbulles injectées.

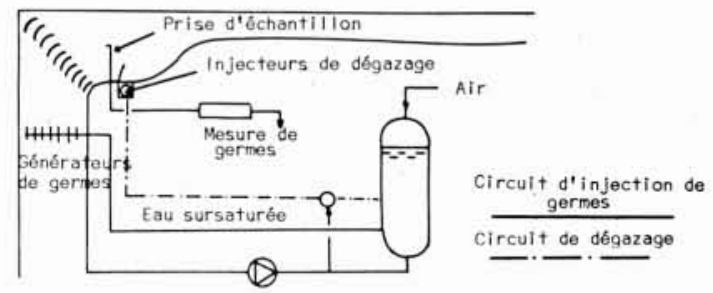

8. Diagramme schématique du système de contrôle de germes et de dégazage du GTH.

\section{Ecrémeur}

$\mathrm{Au}$ débit maximum, $32,4 \mathrm{~m}^{3} / \mathrm{s}$ entrent dans la cuve auxiliaire à une vitesse de $12 \mathrm{~m} / \mathrm{s}$, et l'énergie d'écoulement doit être dissipée sans bruit par friction ou par turbulence. Les éventuelles sources de bruit sont la cavitation aux faibles pressions, l'entraînement des bulles et les fluctuations de l'écoulement.

Il convient de conserver présent à l'esprit que la valeur de $\sigma$ minimale est de 0,16 en surface libre, et que pour une telle valeur, la dissipation turbulente classique entraîne immanquablement une forte cavitation.

Il a été décidé d'utiliser la géométrie illustrée à la figure 9, 125 canaux parallèles ont été construits. Ces canaux ont un angle de divergence très réduit. Leur longueur est de $6 \mathrm{~m}$ et leur profondeur augmente, passant de $10 \mathrm{~cm}$ à l'entrée à $45 \mathrm{~cm}$ à la sortie. Le taux de divergence total est donc de 8 .

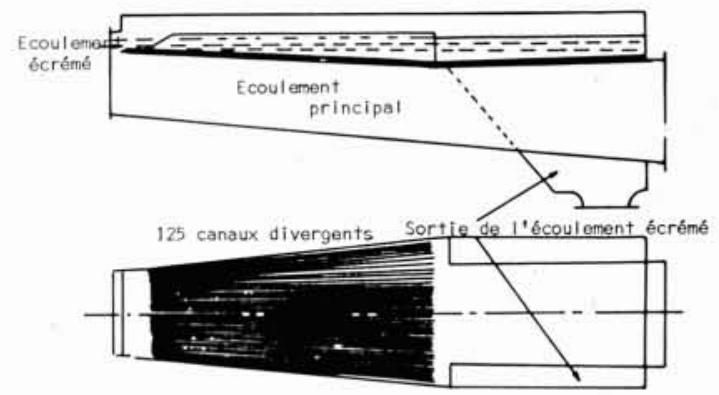

9. Ecrémeur de la veine d'essais en surface libre.

Avec une telle conception, la friction sur les parois est nettement prépondérante et aucune structure fortement turbulente n'est produite. Les entrées sont exemptes de cavitation du fait de leur forme elliptique et de leur pente à $45^{\circ}$. La validité de cette conception, qui évite également toute ventilation d'entrée, a été vérifiée lors d'essais au réel dans le tunnel en surface libre TH2 du CERG.

Une caractéristique intéressante de ce système concerne la hauteur d'eau dans les canaux. Quelle que soit la vitesse, le nombre de Froude à l'entrée du canal est toujours supérieur à 3 , c'est-à-dire que l'écoulement est toujours supercritique. Comme on le sait, un ressaut d'eau est toujours inévitable lorsque la vitesse engendrée par une telle géométrie diminue. Dans cette configuration très spécifique, il est possible de montrer qu'une combinaison donnée de pente et de largeur de canal permet toujours à l'écoulement de rester horizontal, quelle que soit sa vitesse d'entrée et son régime. En d'autres termes, lorsque le niveau aval dans la cuve est conforme aux conditions optimales, le ressaut se produit sans variation de niveau et, lorsque la vitesse change, sa localisation se déplace vers l'amont ou vers l'aval, alors que la surface libre reste horizontale.

Outre le fait que la dissipation se produit sans bruit, ce qui a été vérifié expérimentalement, le fait de n'avoir aucune variation de niveau dans le tunnel quelle que soit la vitesse de l'écoulement facilite bien entendu notablement son utilisation.

On notera, pour l'anecdote, qu'un système analogue peut être utilisé pour éviter la cavitation sur les évacuateurs de crue de barrage ou les sorties de grandes vannes. On peut même envisager de supprimer tout bassin dissipateur d'énergie dans certaines configurations particulières de fonctionnement de grands barrages hydroélectriques.

Le comportement de l'écoulement dans les canaux a été testé systématiquement au CERG dans la gamme de vitesses 1,2 à $10,0 \mathrm{~m} / \mathrm{s}$. On a découvert que la variation de niveau dans le canal n'était jamais supérieure à $20 \mathrm{~cm}$. Les premières expériences réalisées au GTH confirment la très bonne homogénéité des écoulements en sortie des canaux dissipateurs et le silence de fonctionnement de l'écrémeur.

\section{Veines d'essai}

\section{Veine fermée}

La VF est illustrée à la figure 10. Ses dimensions d'ensemble sont de $1,14 \times 1,14 \times 6 \mathrm{~m}$. Au débit maximum $\left(26 \mathrm{~m}^{3} / \mathrm{s}\right)$, la vitesse est de $20 \mathrm{~m} / \mathrm{s}$. Le nombre de Reynolds basé sur un diamètre d'hélice typique de $500 \mathrm{~mm}$ est de $10^{7}$. Le nombre de Reynolds basé sur un corps de $5 \mathrm{~m}$ de long est de $10^{8}$. Le $\sigma$ minimum au centre de la section d'essai est de 0,065 . Les fluctuations moyennes de vitesse dans le temps et l'espace ont pu être mesurées sur la maquette à l'échelle $1 / 6$ réalisée au CERG. Elles sont inférieures à $1 \%$. Le niveau de turbulence admissible maximum est également de $1 \%$. Les premières mesures au réel confirment ces valeurs.

Vingt-et-un hublots en plexiglass de $112 \mathrm{~mm}$ d'épaisseur sont installés à intervalle régulier le long des parois et du fond. Pour empêcher une cavitation excessive à la sortie, les parois supérieure et inférieure de la veine ne sont pas parallèles, mais légèrement divergentes. Les 
parois latérales verticales sont toutefois parallèles, étant données les exigences des mesures optiques.

Une caractéristique essentielle de la VF est que la vitesse peut être de $20 \mathrm{~m} / \mathrm{s}$. Par conséquent, elle tend à éliminer les différences de comportement de la cavitation dues à la qualité de l'eau et au nombre de Reynolds de la maquette.
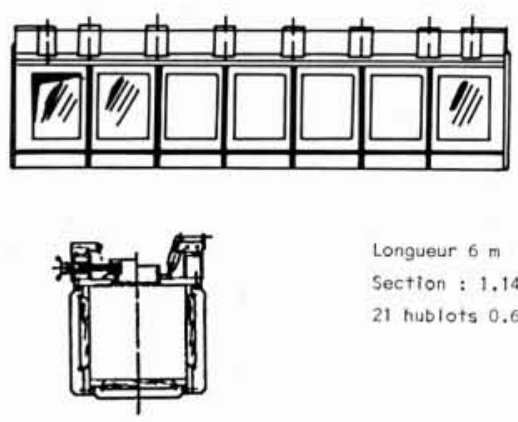

Longueur $6 \mathrm{~m}$

Section: $1.14 \times 1.14 \mathrm{~m}$

21 hublots $0.62 \times 0.62 \mathrm{~m}$

10. Veine fermée.

\section{Veine d'essai en surface libre}

La VSL est différente des conceptions traditionnelles, particulièrement en ce qui concerne l'écrémeur et le système de récupération de pression.

Les tunnels de cavitation en surface libre sont généralement conçus de la manière suivante : le débit provenant de la section d'essai est écrémé par une plaque plane. La partie supérieure de l'écoulement passe sur la plaque, alors que la partie inférieure, qui représente 5 à 10 fois plus d'eau, passe en dessous. La récupération de l'énergie cinétique augmente la pression dans le diffuseur. L'énergie correspondante doit ensuite être perdue au travers de grilles, afin que l'écoulement principal atteigne la cuve aval à une pression identique à celle de la partie écrémée.

Cette conception s'avère satisfaisante pour des petits tunnels de cavitation, dans lesquels toute la puissance cinétique $P$ passant à travers la section d'essai est entièrement dissipée. Si $Q$ représente le débit de l'écoulement et $V$ sa vitesse, la puissance perdue est :

$$
P=Q \times 0,5 \rho V^{2} \text {. }
$$

Les caractéristiques de la VSL du GTH sont les suivantes :

$$
\begin{aligned}
Q_{\max } & =32,4 \mathrm{~m}^{3} / \mathrm{s} \\
V_{\max } & =12 \mathrm{~m} / \mathrm{s} \\
h & =1,35 \mathrm{~m} \\
l & =2 \mathrm{~m} \\
L & =10 \mathrm{~m} \\
\sigma_{\min } & =0,25 \text { au centre de la section d'essai } \\
& =0,16 \text { au niveau de la surface libre. }
\end{aligned}
$$

La puissance perdue au débit maximum serait de :

$$
P=32,4 \times 0,5 \rho(12)^{2}=2,33 \mathrm{MW}
$$

et la puissance installée d'un tel tunnel en surface libre utilisant une conception traditionnelle nécessiterait environ $4 \mathrm{MW}$.

Le GTH comporte un écrémeur pour le débit secondaire et un diffuseur pour le débit principal, comme dans le cas précédent. L'énergie cinétique du flux principal est récupérée comme dans le cas d'une veine fermée, mais un collecteur particulier (fig. 9) est utilisé pour dissiper l'énergie de l'écoulement écrémé. Celui-ci entre dans une cuve intermédiaire d'où il est renvoyé, par une pompe auxiliaire, dans la cuve aval.

Ce concept permet à la plus grande partie de l'énergie de l'écoulement principal d'être récupérée. La puissance totale du système est réduite à 2,0 MW. De même, la pression absolue dans la cuve aval est supérieure et par conséquent, le NPSH d'installation de la pompe principale est bien meilleur qu'avec des solutions conventionnelles.

Le contrôle automatique d'un tel circuit est, bien entendu, plus complexe que le contrôle d'un tunnel de conception traditionnelle. Il suffit de mentionner que la différence de pression entre la section d'essai et la cuve aval dépend de la vitesse d'écoulement et qu'elle doit être contrôlée aux vitesses d'écoulement les plus faibles, à mieux que $1 \mathrm{mb}$.

Les caractéristiques de performance de la VSL sont identiques à celles de la VF, c'est-à-dire, un niveau de turbulence de $1 \%$, ainsi qu'une fluctuation de vitesse et une non-homogénéité moyenne de $1 \%$.

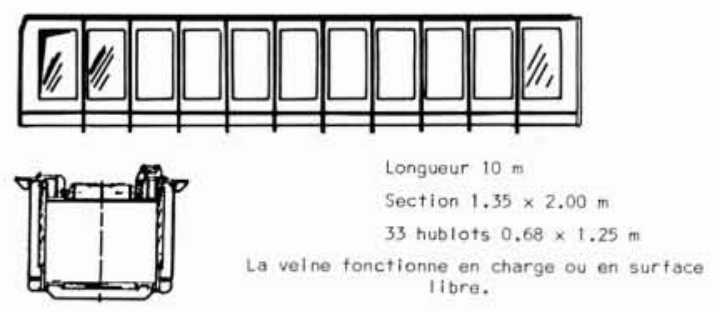

11. Veine d'essais en surface libre.

Les gammes de vitesses sont de 1,25 à $2,55 \mathrm{~m} / \mathrm{s}$ (fluvial) et de 4,75 à $12,00 \mathrm{~m} / \mathrm{s}$ (torrentiel). Le tunnel ne peut être utilisé lorsque l'écoulement est proche du point critique, la vitesse critique étant :

$$
V_{c}=\sqrt{g h}=3,64 \mathrm{~m} / \mathrm{s} .
$$

Du fait en partie de la légère pente du fond $(0,3 \%)$ de la section d'essai, les fluctuations du niveau de l'eau dans la gamme de fonctionnement normal sont inférieures à $1 \%$ de la profondeur totale $(1,35 \mathrm{~m})$. Ces caractéristiques de performance et les autres citées ci-dessus, comprenant les limites de cavitation, ont été vérifiées au CERG sur une maquette à l'échelle $1 / 6$, qui a également servi à définir le système de contrôle automatique et les procédures d'arrêt d'urgence.

Il convient également de remarquer que la VSL peut être utilisée comme veine fermée par mise en place d'un couvercle spécial. 


\section{Système de contrôle du tunnel}

Le système de contrôle du GTH est par nécessité complexe car le fonctionnement de la VSL doit être contrôlé automatiquement. Pour que le fonctionnement du tunnel soit aussi simple que possible, la plupart des fonctions décrites ci-dessus peuvent être réalisées automatiquement, par exemple les réglages de vitesses et de pression dans l'une ou l'autre des sections d'essai, le dégazage, le remplissage du tunnel et la génération de germes. Les procédures d'essai sont, par conséquent, essentiellement automatiques, au moins pour les essais de routine. Les fonctions automatiques comprennent le fonctionnement du vélocimètre laser, l'établissement des courbes $K T$ et $K Q$ et les mesures acoustiques. Le fonctionnement du tunnel est également facilité par trois caractéristiques particulières: (a) les couvercles de la section d'essai peuvent être ouverts et fermés rapidement par des vérins hydrauliques, (b) les maquettes d'essais et les instruments fixés sur le couvercle peuvent être placés et enlevés au moyen de deux ponts de capacité unitaire de $27 \mathrm{~T}$ et (c) les opérations mentionnées ci-dessus peuvent être réalisées sans vider le tunnel, étant donné le maintien d'un niveau constant.

Notons que le passage d'une veine à l'autre peut s'effectuer en moins de 1 journée.

\section{Conclusion et perspectives}

La commande de construction du Grand Tunnel Hydrodynamique sur la base des spécifications élaborées par la Marine française et d'une étude de conception prélimi- naire, a été passée à Alsthom en 1981. Les essais de maquettes et les schémas de construction furent achevés en 1983. Le programme de recherche nécessaire à la préparation des plans comprenait des essais de maquettes au 1/6 des deux sections d'essai, une maquette à l'échelle $1 / 10$ de la cuve aval et des séparateurs d'air. Sept expériences furent également nécessaires pour la vérification des performances du système de contrôle d'air libre et dissout aux conditions de fonctionnement du GTH. Les travaux sur le chantier commencèrent en 1985.

Après les essais préliminaires de recette dont l'achèvement est prévu en 1988, le tunnel sera exploité par les équipes de recherche du Bassin de Carènes, qui utiliseront une instrumentation spécifique pour les activités de $\mathrm{R}$ \& D ainsi qu'un certain nombre de systèmes de mesure comprenant :

- un dynamomètre d'hélices contrarotatives de $250 \mathrm{~kW}$

- un dynamomètre à arbre unique et à faible niveau de bruit de $840 \mathrm{~kW}$

- un système LDV tri-dimensionnel à entraînement automatique

- une balance à six composantes

- un équipement de visualisation comprenant des sources lumineuses, des caméras vidéos et des caméras ultra rapides.

Quatre ordinateurs pour l'acquisition et le traitement des données et pour la calibration des maquettes seront également utilisés pour les essais de routine, automatisés autant que possible. Ces nouveaux systèmes devraient permettre de procéder à des essais avancés dans d'excellentes conditions de fonctionnement et seront utilisés pour la recherche, le développement et les applications industrielles, dans le cadre des programmes nationaux et internationaux.

\author{
Adresses des auteurs : \\ Monsieur $Y$. Lecoffre \\ Chef des Moyens d'Essais, \\ Bassin d'Essais des Carènes, \\ Centre du Val de Reuil \\ Chaussée du Vexin. \\ 27100 Le Val de Reuil (France) \\ Tél. : 38597800 \\ Monsieur $P$. Chantrel \\ CERG-ACB Alsthom \\ voie privée Rhône-Poulenc \\ 38800 Le Pont de Claix (France) \\ Tél. : (16) 76409040 \\ Monsieur J. Teiller \\ ACB Alsthom, \\ Prairie au Duc, BP $2 X$ \\ 44040 Nantes Cedex (France) \\ Tél. : (16) 40411404
}




\section{Références}

[1] M. Visconti et A. Bovis, Le grand tunnel hydrodynamique, ATMA 1983.

[2] Le tunnel hydrodynamique $n^{*} 2$, Rapport interne, Bassin des Carènes.

[3] Y. LeCOFfre, Turbulence et séparation, Société hydrotechnique de France, Conférence, mars 1987.

[4] Y. LeCOFfre, Procedures and Instrumentation for Monitoring Gas Content in Cavitation Test Loops, ASME International Symposium on Cavitation Research Facilities and Techniques, Boston, december 1987.

[5] D. M. Oldenziel, R. H. J. Hansen, A. P. Keller, Y.
LeCOFFre et R. L. VAN ReNesse, Comparison of Instruments for Detecting Bubbles and Particles in Water during Cavitation Studies, 11th IAHR Symposium, Amsterdam, 1982.

[6] Y. Lecoffre, P. Chantrel, J. Teiller, Le grand tunnel hydrodynamique (GTH): France's new large cavitation tunnel for Naval Hydrodynamics Research, ASME Winter Annual Meeting, Boston, 1987, Symposium on Cavitation Facilities and Techniques.

[7] L. BRIANÇON-Marjollet, Couches limites, germes et cavités en interaction : étude physique, Thèse de l'USTMG, Grenoble, octobre 1987.
M. DOREY: Vous avez dit que vous aviez fait des mesures à $1 \%$ près. Vous évaluez à combien la précision des mesures sur le taux de germes?

M. LECOFFRE: Sur le taux de germes, il y a un certain nombre de problèmes qui se posent.

D'abord, on ne peut pas parler globalement d'un taux de germes; on est obligé de réaliser des histogrammes de germes. Il faut définir une sorte de moyenne, qui tient compte de la distribution spatiale des germes dans l'écoulement et d'éventuelles fluctuations temporelles de concentrations.

Du point de vue des précisions de mesures et en ce qui concerne les venturis, il faut se référer à la thèse de Mme BrIANÇONMARJOLLeT, qui utilise la même méthode que celle du GTH.

Je pense qu'à 10 ou $20 \%$ près, on a des mesures fiables en concentration.

$M$. BONNIN : Que coûte en fonctionnement, et surtout en investissement, ce remarquable outil technique?

M. LECOFFRE: Il coûte moins cher au client qu'à la Marine Nationale. Le coût global est de 400 millions de francs. Quant au coût de fonctionnement, disons que les tarifs ne tiennent pratiquement pas compte de l'amortissement du tunnel, et du bâtiment. Par contre, ils tiennent compte de l'amortissement des moyens d'essais et de mesures associés.

M. GRISON : Pouvez-vous détailler les autres moyens de mesures mis à part les germes ?

M. LECOFFRE : Nous disposons d'un certain nombre de moyens de mesures assez intéressants :

- Pour la mesure des vitesses, nous utilisons un laser tridimensionnel qui permet d'avoir accès aux trois composantes de la vitesse. C'est un appareil que nous allons continuer à développer dans le but d'avoir des temps de mesure très courts.

On pense également développer des robots qui seraient utilisés pour faire des mesures en diffusion avant, et dont le rôle est de suivre automatiquement le point de mesure, sachant que l'optique d'émission est située à $3 \mathrm{~m}$ de l'optique de réception. Nous sommes également à l'écoute de tout ce qui se fait de nouveau en holographie et autres méthodes analogues dans ce domaine de la mesure des vitesses.

Pour les mesures de couple et poussée, nous avons actuellement un carter contra-rotatif d'une puissance de $250 \mathrm{~kW}$. On peut entraîner deux hélices tournant en sens contraires, ou faire tourner une hélice simplement dans un sens. C'est un moyen d'essai important, qui est en cours d'essai de recette.
Nous avons également en préparation un carter de $860 \mathrm{~kW}$ entraîné par une ensemble de turbines axiales et qui présente la particularité de ne pas faire de bruit. La machine a un diamètre extérieur de l'ordre de $250 \mathrm{~mm}$. Ce carter est de conception ACBCERG. C'est M. Chantrel qui est chargé de suivre cette question. C'est un outil intéressant et nous pensons éventuellement faire, sur le même principe, la même chose avec un petit carter de $60 \mathrm{~kW}$ environ.

Pour les mesures d'efforts, nous avons une balance à six composantes d'une capacité de 1,5 tonne. Nous avons également en cours de réalisation une balance de paroi que l'on peut déplacer en translation selon deux axes et en rotation.

M. GRISON: Où en êtes-vous en intensimétrie?

M. LECOFFRE : C'est un sujet qui nous préoccupe. Pour l'instant, on n'a pas encore tout à fait fini d'étudier le détail des méthodes de mesure. Il est prévu de réaliser une cuve acoustique : on va entourer la veine d'essais avec un grand réservoir d'eau, ce qui va permettre d'une part, de faire un traitement du signal assez sophistiqué. L'objectif est de réaliser, à partir des mesures ainsi effectuées, un déconfinement du signal. On espère, à partir de la mesure en tunnel, prévoir le bruit rayonné par le prototype.

Une des choses essentielles, avant de lancer l'opération, est de s'assurer que le tunnel est lui-même silencieux. Les premiers résultats d'essais semblent montrer que le GTH est actuellement le meilleur tunnel du monde sous cet angle.

M. DESCLAUX : Vous avez parlé, à propos de la cuve aval et du réservoir, de temps de séjour. Pouvez-vous nous donner un ordre de grandeur des temps de séjour et également du temps de transit d'une particule sur l'ensemble de la boucle?

M. LECOFFRE: Dans une boucle d'essai, la masse de gaz contenue dans les bulles évolue en tous points. Le phénomène de la vitesse, de la pression, de la dimension des bulles, dépend de certains paramètres, comme la turbulence ou la teneur en air dissous.

En fait, tout le tunnel participe au contrôle des bulles. On a cherché à optimiser le GTH pour effectuer le contrôle des germes avec un volume minimal.

M. DESCLAUX: Vous avez cherché à l'optimiser autour d'un chiffre précis ou non?

M. LECOFFRE: Le chiffre dépend du retour des bulles dans la veine. Je crois qu'on ne peut pas répondre avec des chiffres précis ; c'est un sujet relativement complexe qui demande un calcul complet. 